\title{
HP3-RAD: A compact radiometer design with on-site calibration for in-situ exploration
}

\author{
Emanuel Kopp ${ }^{\mathrm{a}}$, Nils Mueller ${ }^{\mathrm{b}}$, Matthias Grott ${ }^{\mathrm{b}}$, Ingo Walter ${ }^{\mathrm{a}}$, Joerg Knollenberg ${ }^{\mathrm{b}}$, Frank \\ Haenschke ${ }^{\mathrm{c}}$, Ernst Kessler ${ }^{\mathrm{c}}$, Hans-Georg Meyer ${ }^{\mathrm{c}}$ \\ ${ }^{a}$ DLR Institute of Optical Sensor Systems, Berlin, Germany; ${ }^{b}$ DLR Institute of Planetary Research, \\ Berlin, Germany; ${ }^{\mathrm{C}}$ Institute of Photonic Technology, Jena, Germany
}

\begin{abstract}
Many processes on planetary bodies are driven by their respective surface energy balance, and while planetary climate is influenced by the dynamics of the atmospheric boundary layer, surface radiation drives the Yarkovksy and YORB effects on small airless bodies. In addition, insolation governs cometary activity and drives the dust cycle on Mars. The radiative flux received and emitted at the surface of solar system bodies is thus a fundamental quantity, which is driven by the reception of solar radiation in the visible wavelength band, while re-radiation primarily occurs in the thermal infrared.

Knowledge of the relevant radiative fluxes enables studies of thermo-physical surface properties, and radiometers to measure surface brightness temperatures have been payloads on many missions.

The HP3-RAD is part of the Heat Flow and Physical Properties Package (HP3) on the InSight mission to Mars. It is a light-weight thermal infrared radiometer with compact design. HP3-RAD measures radiative flux in 3 spectral bands using thermopile detectors. The $120 \mathrm{~g}$ device includes integrated front-end electronics as well as a deployable cover that protects the sensors from dust contamination during landing. In addition, the cover is simultaneously used as a calibration target. The instrument concept as well as its implementation will be described, and special emphasis will be put on technological challenges encountered during instrument development.
\end{abstract}

Potential future improvements of the design will be discussed.

Keywords: HP3, radiometer, on-site calibration, thermal infrared, thermopile array

\section{INTRODUCTION}

The principle of radiometric measurement of the surface brightness temperature with thermopile array sensors is a well proven concept which is applied at many space missions, e.g. at Thermal Mapper of the Multi-Purpose Sensor (MUPUS$\mathrm{TM}^{1}$ ) on Rosetta Philae, MErcury Radiometer and Thermal Infrared Spectrometer (MERTIS ${ }^{2}$ ) on Bepi-Colombo or Rover's Environmental Monitoring Station $\left(G_{T S}-R_{E M S}{ }^{3}\right)$ on Mars Science Laboratory.

The HP3-RAD design has its heritage in mainly in the MASCOT radiometer (MARA ${ }^{4}$ ). It is a redesign of the original MARA concept with special consideration of size, mass and the ability of in-flight calibration. Furthermore the same thermopile sensors are used.

The HP3 instrument measures thermal conductivity of Mars as a function of depth while penetrating up to $5 \mathrm{~m}$ of regolith, then measures the subsurface thermal profile for the remainder of a Mars year.

It measures the heat flux coming from the interior of Mars at the landing site of the InSight mission. Heat flow is a major constraint on models of the current state of Mars' interior and is key to understanding the evolution of terrestrial planets in general.

The top level science requirement of the HP3 radiometer is that the brightness temperature measurements characterize the seasonal variation of subsurface heat flow. The radiometer only adds redundancy in case that the $\mathrm{HP}^{3}$ measurements do not meet their requirements, most critically being the penetration depth.

This paper describes the radiometer design as well as the technological challengers that occurred with respect to the requirements in mass and size. 


\section{RADIOMETER PRINCIPLES}

\subsection{Measurement principle}

The radiometer design is based around IPHT TS $72 \mathrm{~K}$ thermopile sensors in a temperature-controlled aluminum body. The sensors respond to the temperature difference between the junctions of serial thermocouples (the thermopile) where one of the two junctions of each thermocouple is coupled to the thermal infrared radiation of the observed surface via an area with high absorbance (the absorber). If the temperature of the observed surface is different from the sensor temperature, the net radiative flow of heat over the absorber surface results in a temperature difference between the two junctions and therefore a thermoelectric voltage

$$
U_{T C}=S A F
$$

where $S$ is sensor sensitivity with $[S]=\mathrm{V} / \mathrm{W}, A$ is absorber area with $[A]=\mathrm{m}^{2}$ and $F$ is net radiative heat flow density over $A$ with $[F]=\mathrm{W} / \mathrm{m}^{2}$. Further simplifying to the net heat flow over the absorber surface yields:

$$
Q=A F
$$

Sensitivity depends on the thermopile and fillgas of the sensor cap (or lack thereof). In case that the housing of the sensor cap is at the same temperature as the absorber area the heat flow density is given by the integration of thermal emission specific radiance of absorber area and surface over wavelength $\lambda$ and the hemisphere solid angle expressed as inclination $\vartheta$ from optical axis $(\vartheta=0)$ and azimuth $\varphi$

$$
Q=A \int_{0}^{2 \pi} \int_{0}^{\frac{\pi}{2}} \int_{0}^{\infty} \tau(\lambda) f(\vartheta) \varepsilon(\lambda, \vartheta, \varphi) \varepsilon_{\text {ref }}(\lambda)\left[B\left(\lambda, T_{\text {surf }}(\vartheta, \varphi)\right)-B\left(\lambda, T_{\text {ref }}\right)\right] \cos \vartheta d \varphi \sin \vartheta d \vartheta d \lambda
$$

where $\tau$ is sensor cap filter window transmittance, $f$ is field of view relative sensitivity, $\varepsilon$ is surface emissivity, $\varepsilon_{\text {ref }}$ is absorber emissivity, $B$ is the Planck-function, $T_{\text {surf }}$ is surface temperature and $T_{\text {ref }}$ is temperature of the sensor.

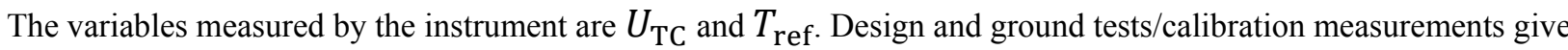
knowledge of all other factors with exception of $\varepsilon(\lambda, \vartheta, \varphi)$, which must be constrained independently.

In order to calibrate the instrument, the voltages when observing a range of known temperatures and a known emissivity, ideally a blackbody $\varepsilon(\lambda)=1$, are recorded. This gives numerical values for the calibration function defined by:

$$
\Gamma\left[U_{T C}\left(T_{\text {surf }}, \varepsilon=1\right)\right]=T_{\text {surf }}
$$

The calibration function $\Gamma$ can be represented by an appropriate fit or by linear interpolation between sufficiently dense samples. To derive a surface temperature from measured voltages $U_{T C}$ it is useful to assume a constant emissivity $\varepsilon_{\text {eff }}$ which approximately fulfills:

$$
\varepsilon_{\text {eff }} \int_{0}^{\infty} \tau(\lambda)\left[B\left(T_{\text {surf }}, \lambda\right)-B\left(T_{\text {ref }}, \lambda\right)\right] d \lambda=\int_{0}^{\infty} \varepsilon(\lambda) \tau_{X}(\lambda)\left[B\left(T_{\text {surf }}, \lambda\right)-B\left(T_{\text {ref }}, \lambda\right)\right] d \lambda
$$

Then the estimated surface temperature is

$$
T_{\text {est }}=\Gamma\left[\frac{U_{\mathrm{TC}}}{\varepsilon_{\mathrm{eff}}}\right]
$$


The accuracy of $T_{\text {est }}$ depends on the knowledge of the target emissivity spectrum $\varepsilon(\lambda)$. The assumption of a constant value for $\varepsilon_{\text {eff }}$ can only be exact for spectrally flat surfaces $(\varepsilon(\lambda)=$ const.), otherwise there is a dependence on $T_{\text {surf. }}$

\subsection{Expected surface emission}

The Mars Exploration Rovers provide data of TIR surface emissivity from MTES data. Data from various targets on the two explored regions Gusev Crater and Meridianum Planum indicate that the expected surface emissivity in the 8-14 $\mu \mathrm{m}$ region is approximately 0.97 with likely variation of \pm 0.02 (See Fig. 1). The surface soil of Mars consists to large amounts of aeolian sediment and forms a global unit ${ }^{5}$ Emissivity of the soil can be expected to be similar in the all regions of the Planet. The top layer of the soil often consists of very fine, light toned dust. Large basaltic rocks (e.g. Humphrey in Gusev) show a diverging emissivity towards their top and in abraded areas, but are more similar to the soil emissivity close to the surface. Soil disturbed by the rover tracks appears darker visually ${ }^{6}$.

Gusev bright soil

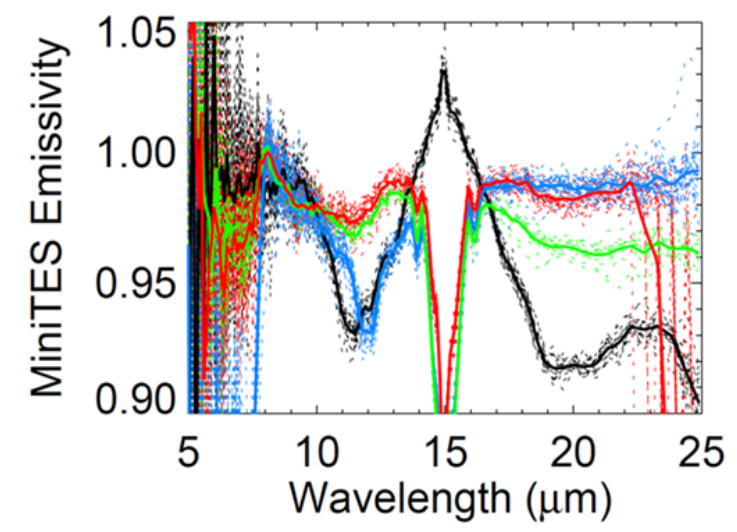

Expected emissivity in the $8-14 \mu \mathrm{m}$ bandpass: $0.97 \pm 0.02$

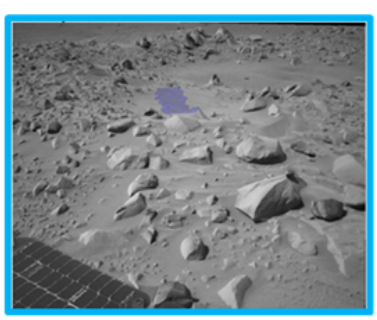

Gusev disturbed soil

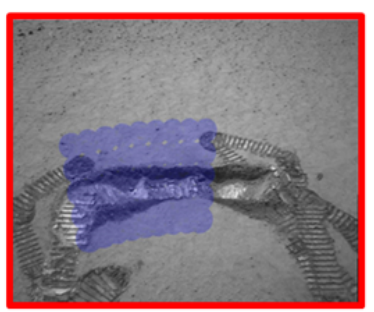

Gusev basalt (Humphrey)

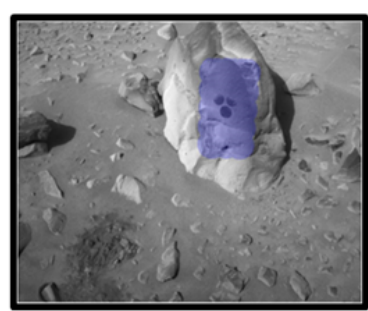

Meridianum disturbed soil

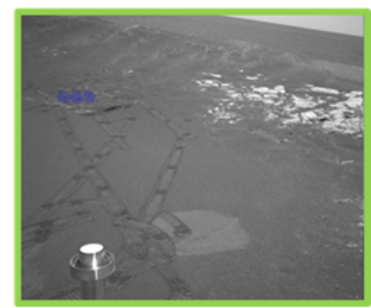

Figure 1 MTES spectra (color coded with data variation as dotted plots) and images indicating location of the corresponding targets

\subsection{Expected surface temperature}

The expected surface temperature $T_{\text {surf }}$ as function of local time and season depends primarily on the landing site latitude, thermal inertia $I$, visible albedo $\alpha$ and atmospheric dust opacity $\tau_{\text {dust. }}$ Local slope and slope dip azimuth and to some extent, surface roughness, that is variation of local slope within the FOV are relevant for analyzing the radiometer data but do not strongly affect the range of expected temperatures. Diurnal temperature cycles for extreme cases of the parameters are calculated using $\mathrm{KRC}^{7}$ presented in Fig. 2 for the warmest and coldest season of the year. The curves are plotted for all landing site candidates but the differences are negligible. The minimum expected temperature is 150-160 $\mathrm{K}$, maximum temperature is $310 \mathrm{~K}$. As nominal case for the following calculations we select $4.96^{\circ} \mathrm{N}$ latitude, thermal inertia $\mathrm{I}=150 \mathrm{TIU}$, albedo $\alpha=0.25$ and $\tau_{\text {dust }}=0.3$. 


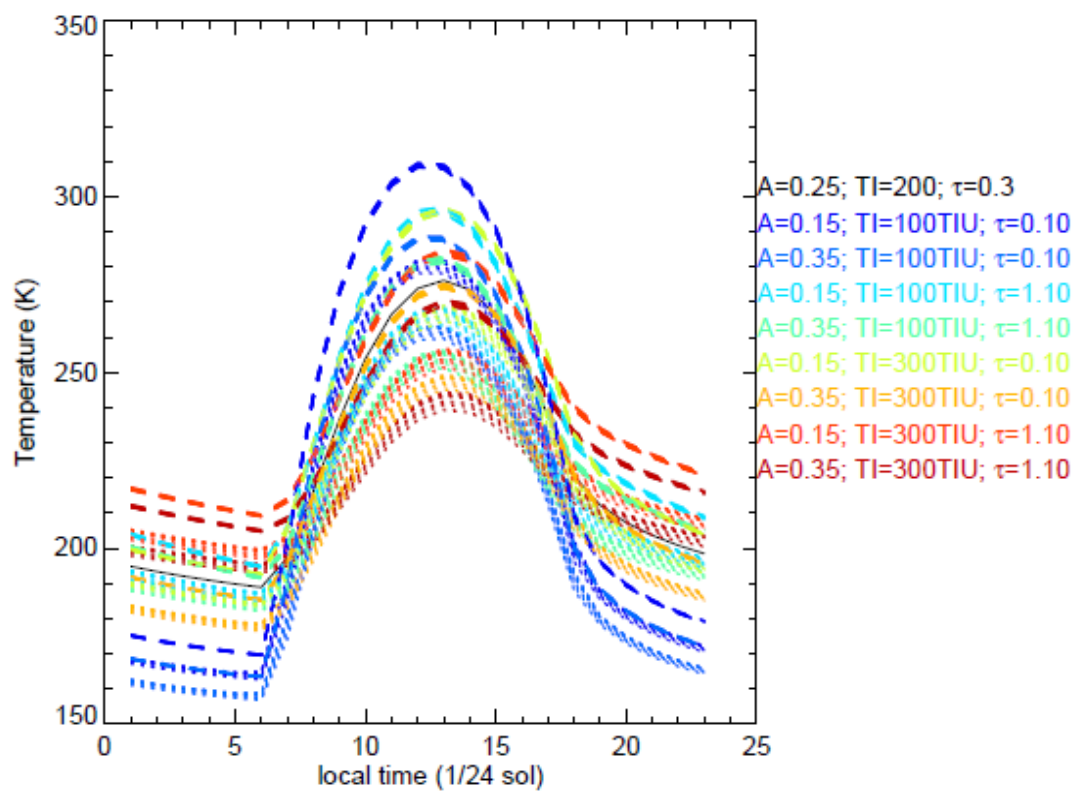

Figure 2 Range of diurnal temperature cycles that might be encountered.

\subsection{Radiometer operations}

Foremost the radiometer needs to measure the annual variation of the daily average temperature. To separate the annual from diurnal variation the diurnal cycle is resolved by measuring at 24 evenly separated local times. This measurement is assume to occur regularly once every $15^{\circ}$ of Ls, i.e. on 24 evenly separated occasions (seasons) over the course of 1 Martian year. The instrument temperature $T_{\text {ref }}$ is relevant for measurement accuracy and power consumption. A very accurate measurement could be achieved by changing $T_{\text {ref }}$ until $U_{T C}=0$ and thus $T_{\text {ref }}=T_{\text {surf }}$. However since the instrument can only control temperature via heaters and the lowest nighttime temperature are below min ops. temperatures, a $T_{\text {ref }}$ setpoint above environmental temperature is required. Since every temperature setpoint needs to be calibrated, we assume only day and night time setpoints of $300 \mathrm{~K}$ and $240 \mathrm{~K}$, respectively. The radiometer observes in three different spectral filters but here only the primary filter, a bandpass from 8-14 micron is considered.

\subsection{Error from emissivity on surface temperature estimate}

The surface temperature estimate $T_{\text {est }}$ with error bars over such a diurnal cycle measurement derived from thermopile voltage $U_{\mathrm{TC}}$ with an error equivalent to $850 \mathrm{nV}$ (equiv. to $4 \mathrm{~K}$ at $150 \mathrm{~K}$ brightness temperature) while observing a surface with emissivity 0.97 is plotted in Fig.3. To illustrate the effect of the uncertainty in surface emissivity, the assumed constant surface emissivity $\varepsilon_{\text {eff }}$ is either too low ( 0.95 , in blue) or too high ( 0.99 , in red). 


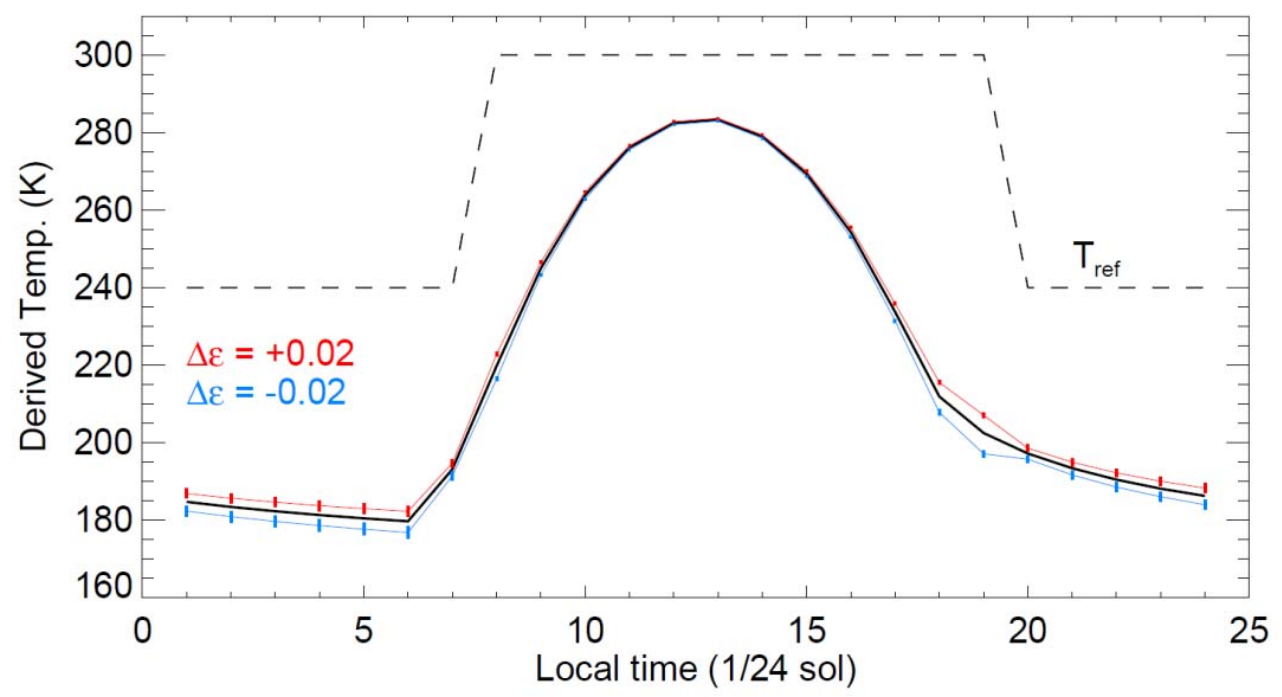

Figure 3 Simulated diurnal cycle of measurements with derived temperature based on incorrect assumption on emissivity. Red and blue error bars indicate thermopile voltage measurement error corresponding to $4 \mathrm{~K}$ at $150 \mathrm{~K}$.

\subsection{Constraint on subsurface heat flow}

The daily average temperature for different seasons is shown in Fig. 4 for one realization of random noise with a normal distribution. A misestimate of surface emissivity primarily introduces an offset in the annual temperature wave, shape and amplitude are mostly preserved. Assuming that the thermal conductivity of the subsurface is known, shape and amplitude constrain the seasonal variation of subsurface heat flow. Heat flow is calculated by using a forward model solving the heat transport equation using a 1D finite differences scheme with the estimated daily average temperature as upper boundary condition, heat flow zero as lower boundary condition $(10 \mathrm{~m}$ depth) and the estimated annual temperature as initial temperature. The estimated annual cycle is repeated 20 times to allow the thermal wave to overcome the constant initial conditions.

Fig. 5 shows the deviation of the reconstruction of seasonal heat flow from the true value, for a depth of $2 \mathrm{~m}$ in a soil with thermal conductivity and density as in the nominal case assumed in $\mathrm{HP}^{3}$ performance simulation.

These figures show that a random measurement error in the thermopile voltage equivalent to $4 \mathrm{~K}$ at $150 \mathrm{~K}$ brightness temperature is less significant than the possible error from the unknown emissivity. Improved instrument performance will not improve the reconstruction of heat flow.

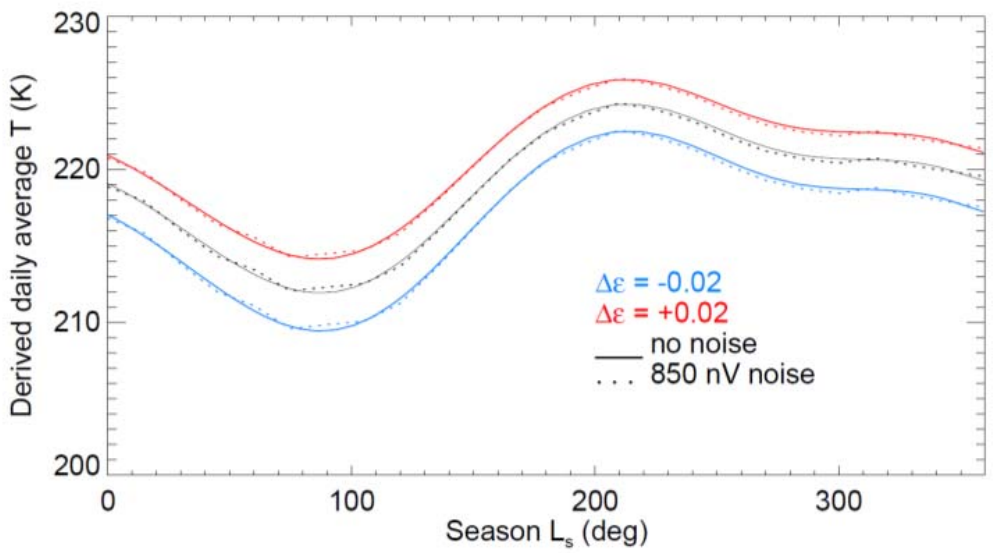

Figure 4 Effect of random noise and emissivity misestimate on measured annual cycle of daily average temperature. 


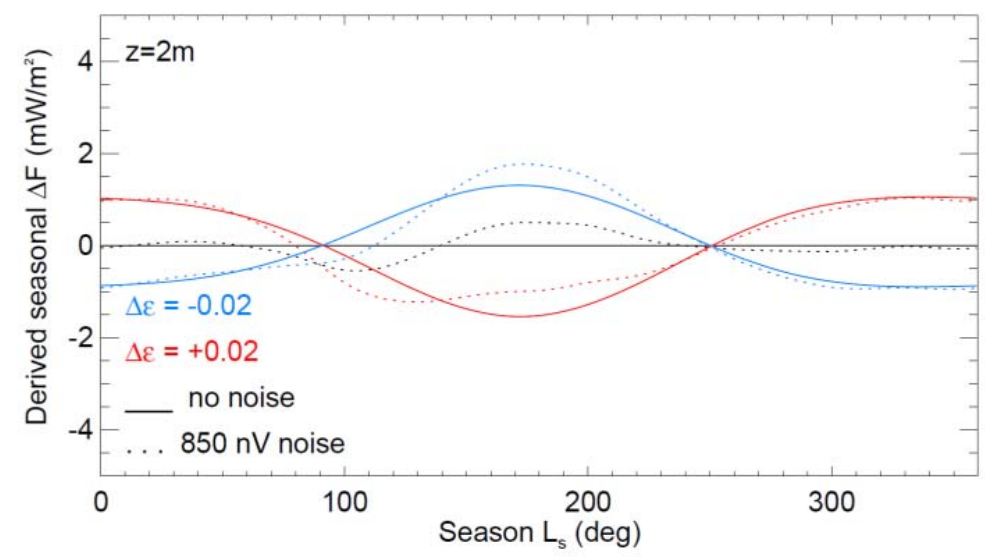

Figure 5 Deviation of the reconstruction of heat flow at $2 \mathrm{~m}$ depth from the true value.

The assumption of only random measurement errors may be optimistic, e.g. in case the sensors filter windows are partially obscured by dust. This can be parameterized by an uncorrected reduction of sensor sensitivity over time. 1\% degradation corresponds to $4 \mathrm{~K}$ difference in brightness temperature at a surface temperature of $150 \mathrm{~K}$. Fig. 6 and Fig. 7 show the effect resulting of $1 \%$ sensitivity loss over the course of 1 Martian year on the daily average temperature. The estimated daily average temperature deviates from the true daily average temperature for approximately $1 \mathrm{~K}$ at the end of the year.

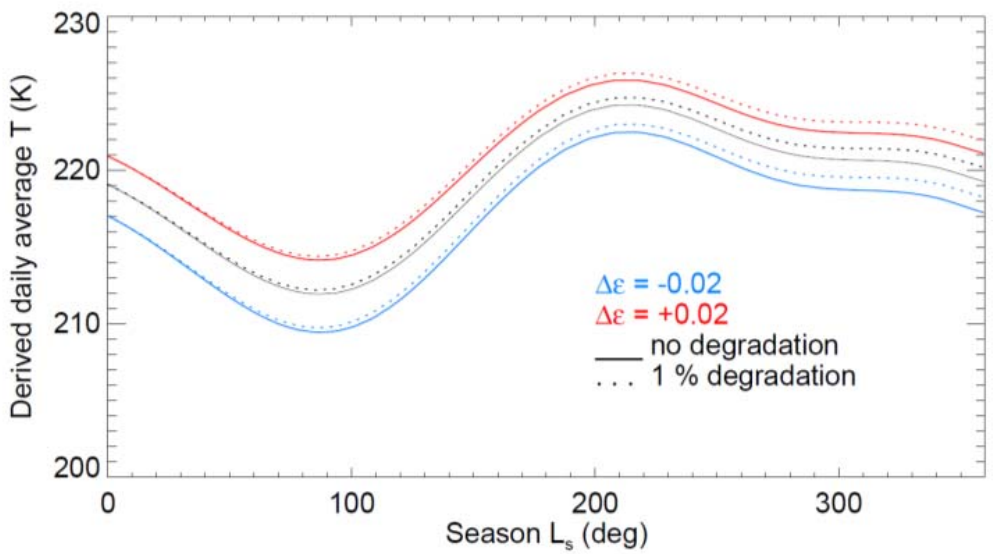

Figure 6 Estimated daily average temperature as a function of emissivity misestimation and sensor degradation. 


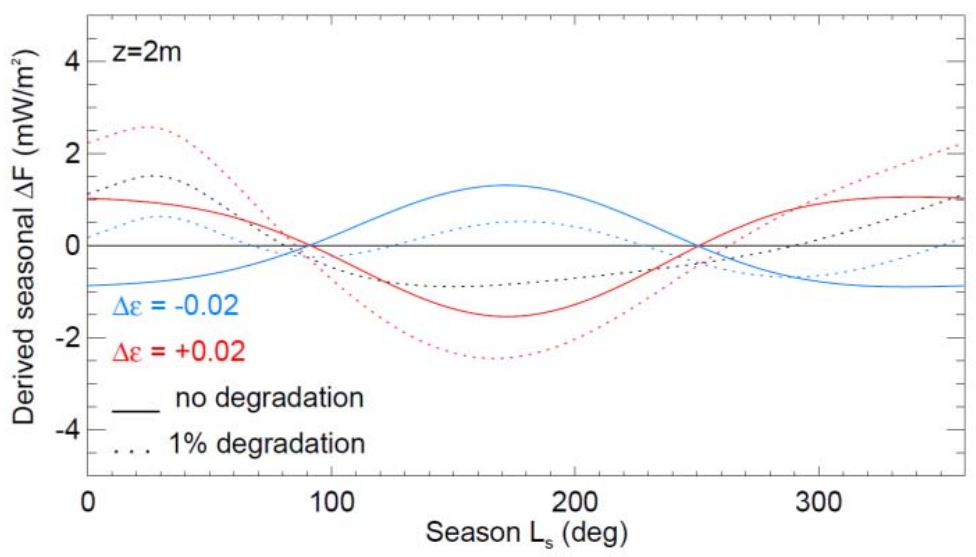

Figure 7 Error of reconstructed heat flow at $2 \mathrm{~m}$ depth due to sensor drift.

The resulting reconstruction of heat flow is precise to within $3 \mathrm{~mW} / \mathrm{m}^{2}$ at $2 \mathrm{~m}$ depth and again error from emissivity is of the same magnitude.

\section{RADIOMETER DESIGN DESCRIPTION}

\subsection{Instrument architecture and key design parameters}

The HP3 Radiometer is a temperature controlled instrument with 6 thermopile sensors in pairs equipped with 3 different spectral filters $(8-14 \mu \mathrm{m}, 16-19 \mu \mathrm{m}$ and 7.8 to $9.6 \mu \mathrm{m})$. The sensors are co-aligned in 2 groups of three sensors. The angle between the two resulting FOV centrelines is fixed by design to $\pm 15^{\circ}$ relative to the normal of the back cover plane.

Its main operations modes are:

- $\quad$ Measure diurnal cycle at least once every $15^{\circ}$ of Ls, once every hour for one sol.

- Measure if possible every sol on four fixed local times.

- $\quad$ Measure for flexible times/periods/sampling frequencies.

- $\quad$ Calibrate the instrument before diurnal cycle measurement.

To meet measurement accuracy requirements the radiometer has a heated and temperature controlled sensor body.

To ensure redundancy, each three sensors with different filters are analyzed by one AD converter. The sensors on one AD converter have the same FOV.

For inflight calibration the Sensor Cover Unit includes a foil heater and a PT100 temperature sensor.

During launch, cruise and EDL the Sensor Cover is closed. After landing and initial calibration the Sensor Cover over is opened. Later calibration uses a mixed pixel calibration approach. The closed Sensor Cover is also a dust protection. To minimize the amount of active electronics parts exposed to low temperatures the electronics is splitted in two parts, one located in the radiometer and one in the back-end-electronics (BEE) on the InSight lander.

\subsection{Electronics}

Two ADS1258 AD converter are used in the radiometer to read out and convert the signals from the thermopile sensors and the PT100 temperature sensors used for temperature control of the sensor body and the sensor cover. An offset 
correction is foreseen for all $\mathrm{AD}$ converter measurement channels. An additional PT1000 temperature sensor, used to monitor the radiometer electronics board temperature is directly connected to the back-end-electronics.

All other components, as temperature control, ADC commanding and data processing are located in the back-endelectronics.

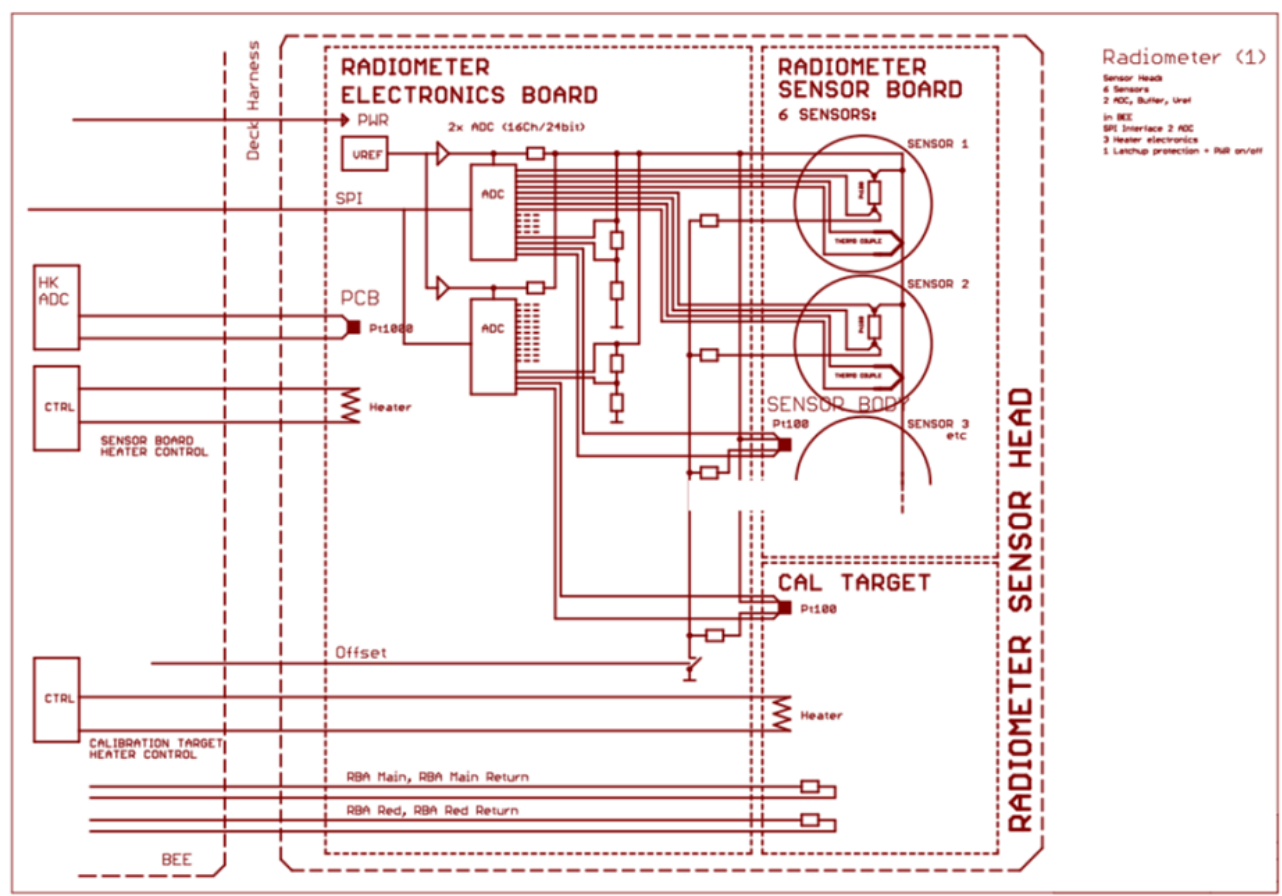

Figure 8 Radiometer electronics block diagram showing the radiometer sensor assembly and the back-end-electronics

\subsection{Mechanical design}

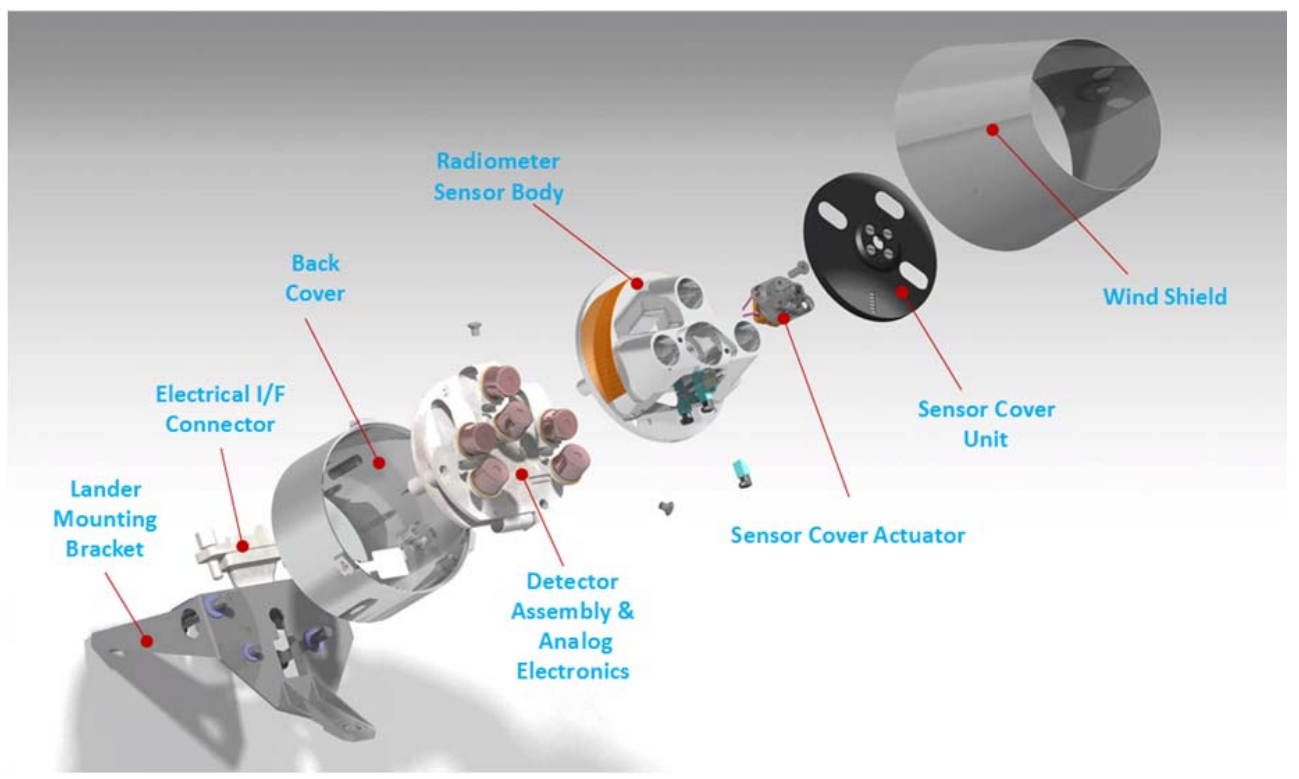

Figure 9 Radiometer sensor head structure and main components 
The radiometer sensor head assembly and its different components are shown in Fig. 9. The following explanations give a more detailed description of selected important parts.

The Lander Mounting Bracket is the mechanical interface between lander and radiometer. It also ensures the thermal isolation of the Radiometer. It consists of a Ti6Al4V-alloy which is processed with selective laser melting technics. This allows a very lightweight and stabile structure design that cannot be realized with conventional manufacturing methods. The mounting bracket interface to the radiometer backplane is at an angle of $40^{\circ}$ relative to the lander deck. The two FOV centerlines are on a plane perpendicular to the lander deck and have an angle relative to the backplane normal of $\pm 15^{\circ}$, and thus $55^{\circ}$ and $25^{\circ}$ relative to the lander deck or the XY plane.

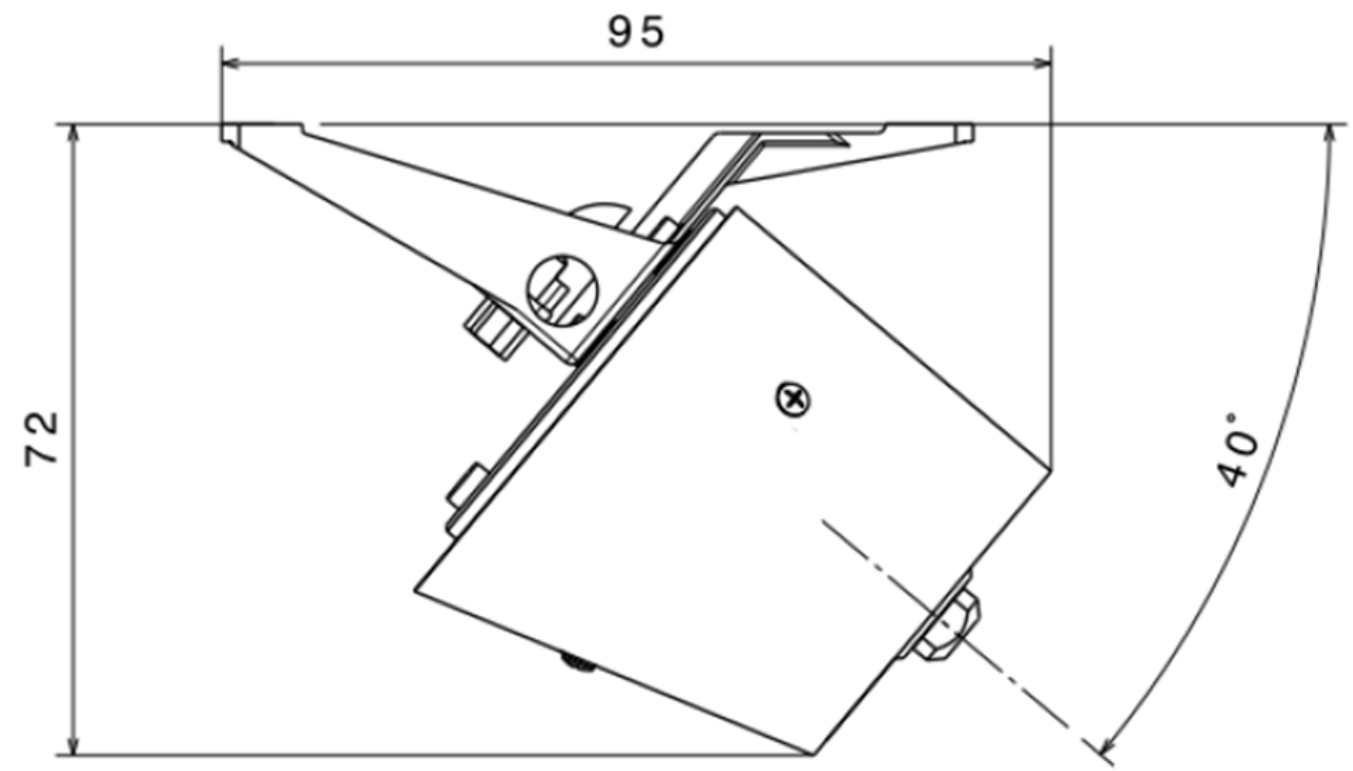

Figure 10 Radiometer sensor head dimensions and mounting orientation

The radiometer sensor body is the aluminum body for the thermopile sensor housing. It is temperature controlled by a 4.5W Capton flex heater at the aluminum body and a PT1000 temperature sensor inside the body. The heater and the sensor are connected directly to the back-end-electronics (BEE). The sensor body heater is also used to heat up the electronics to its minimal operation temperature of $-40^{\circ} \mathrm{C}$ before switching on the radiometer electronics. The thermopile aperture inside the aluminum body is coated with "fractal black".

In order to prevent mechanical failure due to dust contamination, the outer hull of the aperture is designed to have as less surface as possible between dust cover and sensor body.

Due to weight constrains the initial design of the sensor body was very light weight. Tests with a first prototype and thermal simulations showed high temperature gradients of up to $5 \mathrm{~K}$ inside the sensor aperture of the body. Therefor the sensor body was redesigned with a more massive structure on the base of the sensor mounting holes. In addition the position of the sensor body heaters was adapted to the new design. 


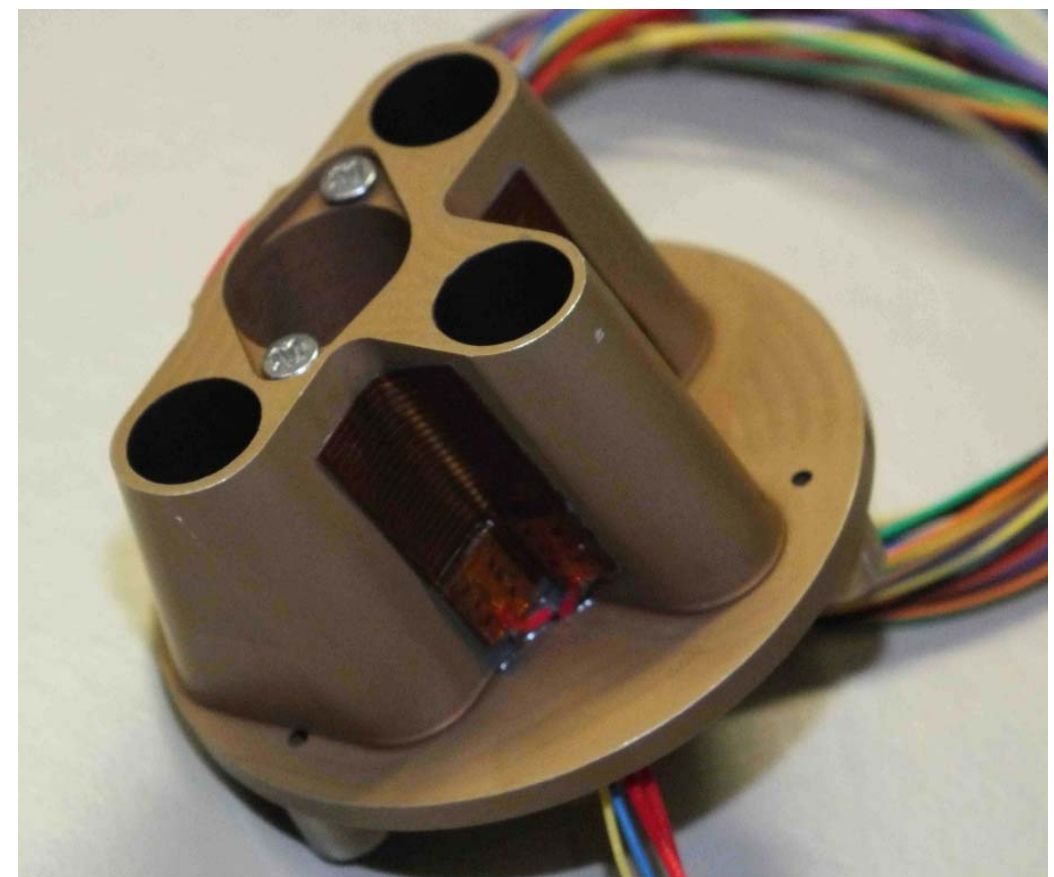

Figure 11 Radiometer sensor body: initial design with heaters attached alongside of sensor apertures

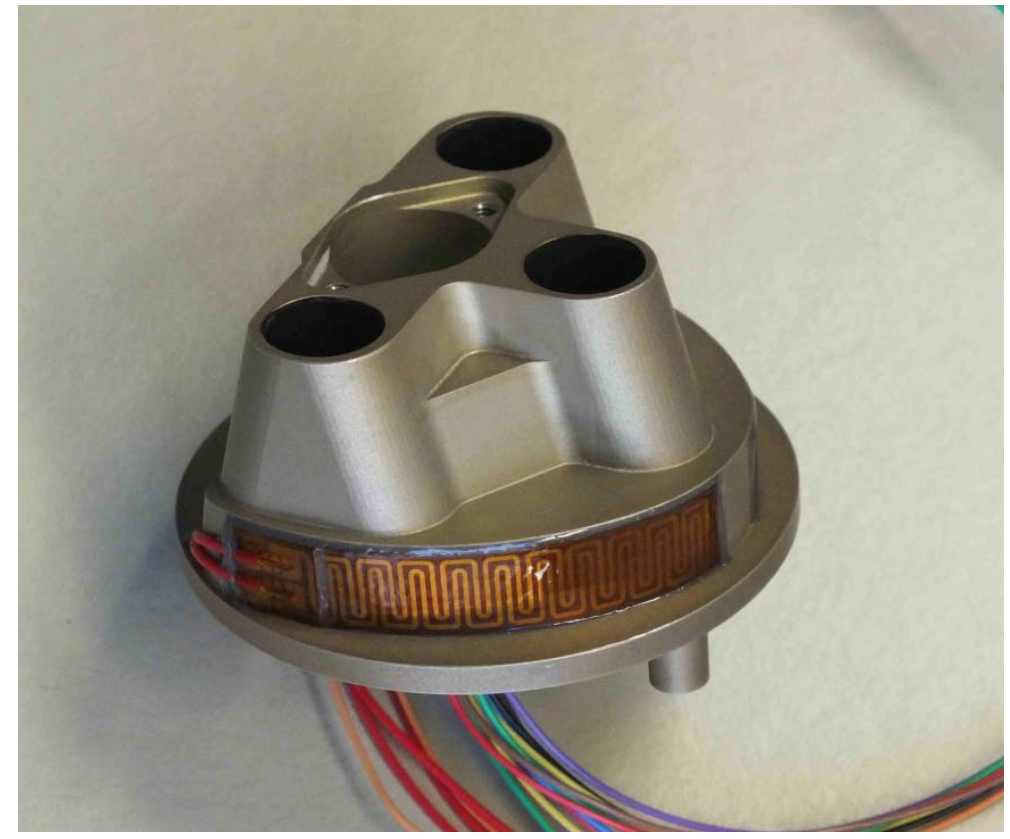

Figure 12 Radiometer sensor body: final design with heaters attached to structure base

The sensor cover is heated from top side with a $2 \mathrm{~W}$ heater foil and has "Kepla Coat" coating on the bottom side. The target is temperature controlled and is used for thermopile sensor calibration (mixed pixel calibration) and as a dust cover during landing for sensor body aperture. 


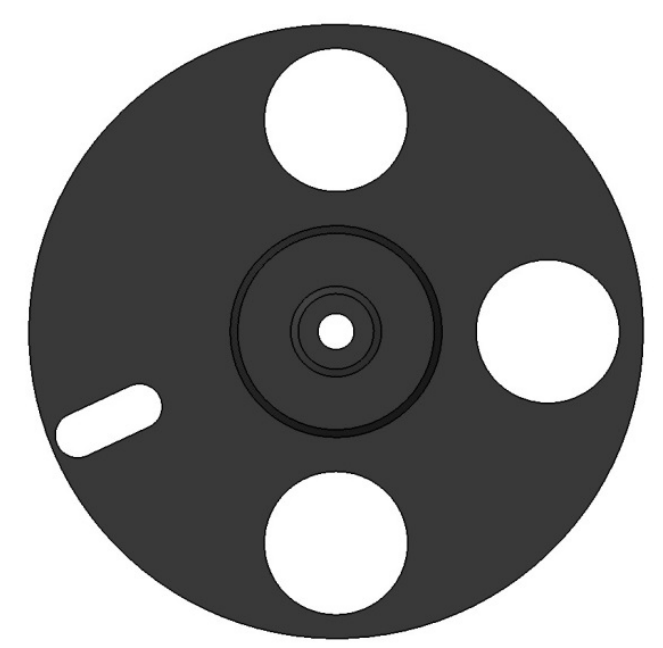

Figure 13 Sensor cover: original design with circular aperture. No cover area in the FOV of the sensor when opened

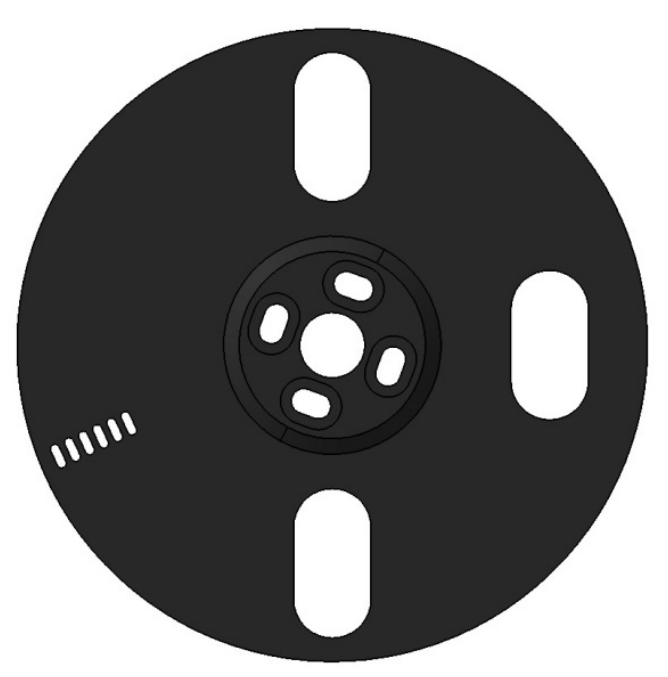

Figure 14 Sensor cover: after redesign with oval aperture for mixed-pixe-calibration

The rotation blade actuator (RBA) is a one-time spring actuator secured with a nylon thread that is wound around a heating resistor. The actuator can be released by heating up the resistor to melt the nylon thread. It opens the Sensor Cover Unit after landing and initial calibration of the instrument. Once released the cover stays open and can't be closed again. The RBA heating resistor is fully redundant.

In the initial design the rotation blade movement was realized with a DC motor and had the capability to sequentially open/close the cover for measurement and calibration. It had to be replaced by the RBA after prototype tests showed high, uncontrolled power dissipation from the motor into the sensor body (description in Sect. 3.1)

The wind shield is the outer shell of the radiometer consisting of gold coated stainless steel. It should reduce thermal losses and protect the electronics and sensor assembly from dust. 


\subsection{Thermopile sensor}

The sensors used in the radiometer are the TS-72K thermopile sensors from IPHT (Institute of Photonic Technology). It is a miniaturized multi-junction thermopile consisting of 72 n-bismuth-antimony/p-antimony (Bi0.87Sb0.13/Sb) thermocouples, with a centrally located hot junction (active) area of $0.2 \mathrm{~mm}^{2}(0.5 \mathrm{~mm}$ diameter) as shown in Fig. 15 . The Seebeck coefficient of each of the thermocouples is $135 \mu \mathrm{V} / \mathrm{K}$. The sensor measurement signal is generated by the temperature difference between the cold and hot junction of the sensing area. To determine the sensor signal, a PT100 temperature sensor measures the cold junction temperature as a reference.

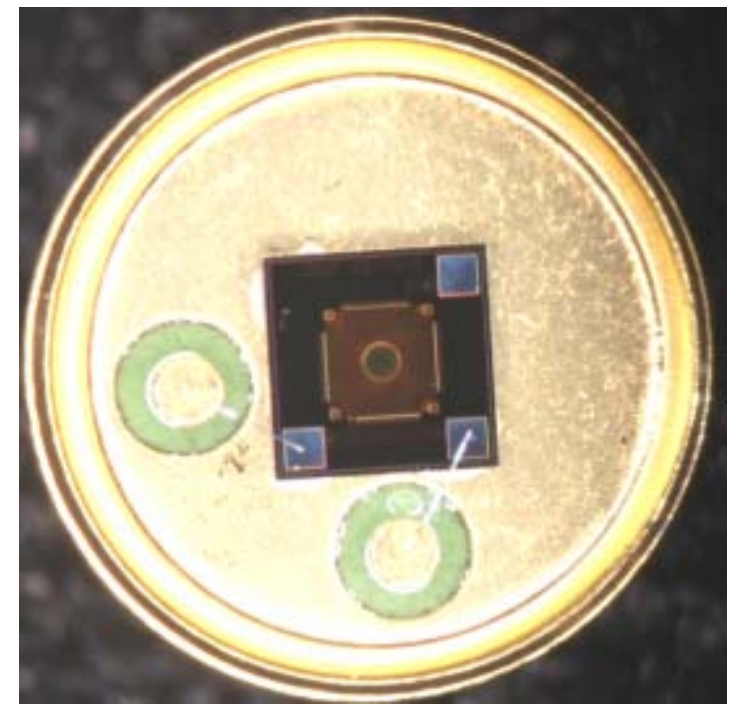

Figure 15 TS-72K thermopile structure base

The thermocouple assembly is accommodated in a TO-39 housing with a laser cut aperture hole on top and black coating on the inside in order to reduce stray-light. Each sensor element is hermetically sealed under Krypton fillgas atmosphere. This is a customization of the standard sensor specification. Normally Xenon which has a higher sensitivity as Krypton is used as fillgas. Since the lowest expected temperature on Mars is about $-125^{\circ} \mathrm{C}$ and Xenon has a condensation point of $165 \mathrm{~K}$ it could condensate under cold Mars conditions. Therefor Krypton with a condensation point of $121 \mathrm{~K}$ was selected for the radiometer sensors. As a last step, the selected spectral filters, as described in Sect. 2.5 are glued to the aperture holes of each sensor.

Fig. 16 shows the six TS-72K sensors soldered to the electronics board. The solder areas of the sensors are connected via flex boards to the main electronics board. 


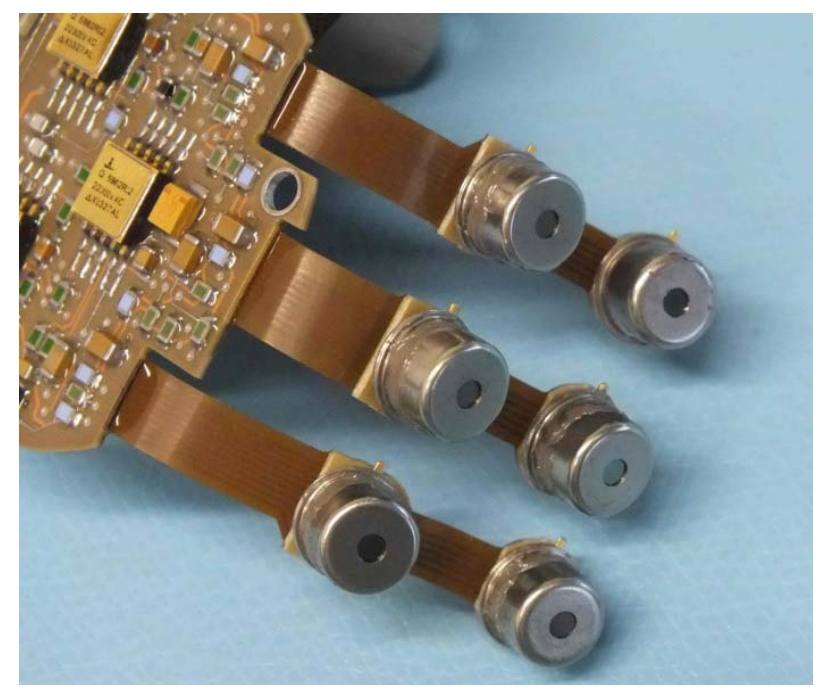

Figure 16 TS-72K thermopile sensors soldered to the radiometer electronics board

\subsection{Spectral filters}

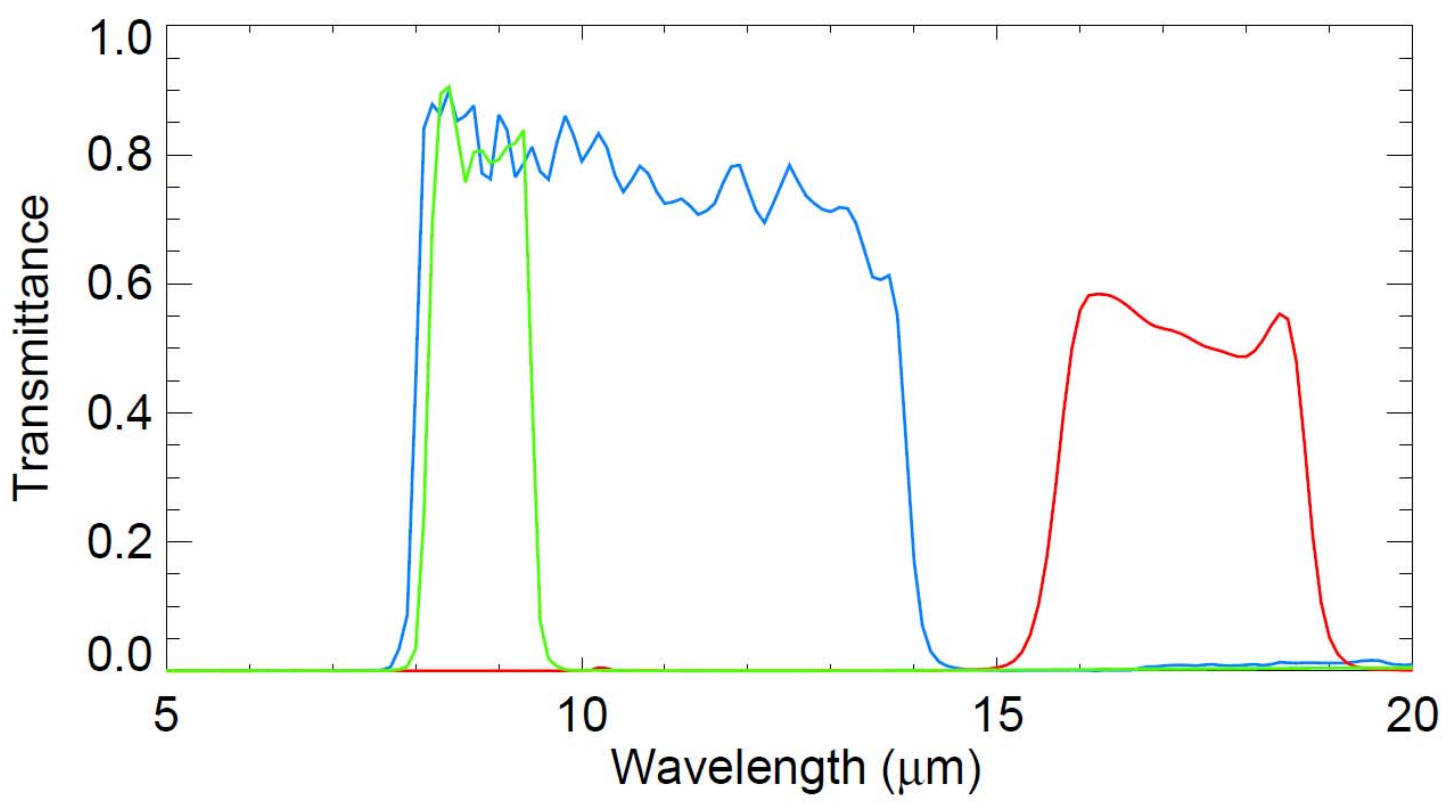

Figure 17 Transmittance of the three spectral filters multiplied with the spectral absorbance of the thermopiles. Blue: 8-14 $\mu \mathrm{m}$ bandpass, green: 7.8-9.6 $\mu \mathrm{m}$ bandpass, red: 16-19 bandpass.

The radiometer measures surface thermal emission which at Mars temperatures has its maximum in the range between $10 \mu \mathrm{m}$ (day temperatures) and $17 \mu \mathrm{m}$ (night temperatures). Reflected sunlight can be a significant contribution up to 3 $\mu \mathrm{m}$. Resolution of the brightness temperatures improve with the width of the spectral filters. We will use 3 different spectral filters in 6 sensors, two of which will be identical with the REMS GTS filters. Sensors are numbered A1, A2, 
B1, B2, C1, C2, where the letter indicates filter and the numeral the FOV position with 1 being the inboard and 2 the outboard. Filters A are both a bandpass 8-14 $\mu \mathrm{m}$, well suited for both day and night temperatures. Filters B are both a bandpass $16-20 \mu \mathrm{m}$, inferior in temperature resolution to the 8-14 $\mu \mathrm{m}$ filter both day and night, but useful for spectral deconvolution of inhomogeneous temperatures in the FOV. Filter transmittance multiplied with thermopile absorbance is plotted in Fig. 17.

\section{INSTRUMENT ON-SITE CALIBRATION}

\subsection{Initial approach for radiometer calibration}

The radiometer operation as initially designed is based on sequential open/ close operation of the sensor cover unit for frequent calibration of the detector signal by fully closed aperture and target measurements by fully opened aperture. This was provided by turning the sensor cover unit of an angle of $45^{\circ}$ with a DC motor without gear powered to turn against mechanical end stop for opening. Reverse powering closes the sensor cover unit and the closed position is kept by the spring effect of the cables connecting the cover heater and temperature sensor to the system.

Since the motor operating in contrast to a bi-stable rotary solenoid is emitting power in the opened position this has to be taken into account for the radiometer sensor body temperature control which gives the reference of the radiative scientific measurements.

According the concept a flight representative prototype has been built and tested according the relevant environment conditions. It turned out that the motor emissive heat which was assumed to be $10 \%$ of the overall heater power of the radiometer conditioning heater system is instead about $50 \%$ which is a permanent instable disturbance source during scientific measurements. This is due to updated thermal analysis and operation scenarios and cannot be compensated by the control electronics (back-end-electronics (BEE) radiometer heater control).

As stabilized temperature conditions of the radiometer body are essential for the measurement accuracy a concept change eliminating the disturbing heat of the DC motor has to be foreseen. Given that a space qualified bi-stable solenoid or a motor extension by gears of the required size is not available the system was changed to a permanent-open Rotor Blade Actuator (RBA) radiometer which is just closed for launch and cruise to Mars, landing and for initial calibration. Therefore the calibration is performed such that in open position a part of the aperture is permanently covered (25\%) and the measurement is following a mixed-pixel strategy. Consequently the DC motor is totally eliminated and replaced by the Rotor Blade Actuator (RBA).

\subsection{Calibration measurements after landing}

The capability to check the on-ground calibration after landing is important to ensure precision over the mission. The greatest risk to the on-site calibration is the contamination of the filter windows by dust, resulting in what is effectively a reduction in the sensitivity of the sensors.

Greatest risk of dust contamination is during landing, at which point the dust cover is still in closed position. Before opening the dust cover a calibration will take place using the same procedure as the later calibrations, however without influence of the temperature of the surface.

After opening the dust cover, calibration should preferably be performed at night when the thermal emission of the surface is small. Mars night time surface temperature at a given site is mostly a function of surface thermal inertia and atmospheric dust opacity ${ }^{3}$. However, even without exact knowledge of thermal inertia and dust opacity ${ }^{3}$ but with knowledge of two surface temperatures at two different times, the surface temperature in the time between is constrained to better than $\Delta \mathrm{T}_{\text {Surf }}=2 \mathrm{~K}$.

Thus given two measurements of surface thermal emission flux, the thermal emission flux in between can be interpolated with an uncertainty corresponding to $\Delta \mathrm{T}_{\text {Surf }}=2 \mathrm{~K}$. Thus if the calibration measurements are bracketed by normal measurements of the surface temperature with sensor cover and body at the same temperature, the surface contribution to the calibration measurements can be extrapolated from the thermopile voltage at the normal measurements and subtracted from the calibration measurements. 


\subsection{Mixed pixel calibration}

The dust cover is redesigned with reduced aperture so that some part of the cover is permanently in the FOV after the one time opening mechanism has been operated (see Fig. 18).

Since the dust cover now contributes to the thermopile voltage during measurements, its temperature needs to be controlled via the heater at all measurements. Preferably the dust cover is kept at the same temperature as the body however it is also possible to choose a lower temperature, provided that the difference between body and dust cover is the same for all measurements including ground and flight calibration measurements.

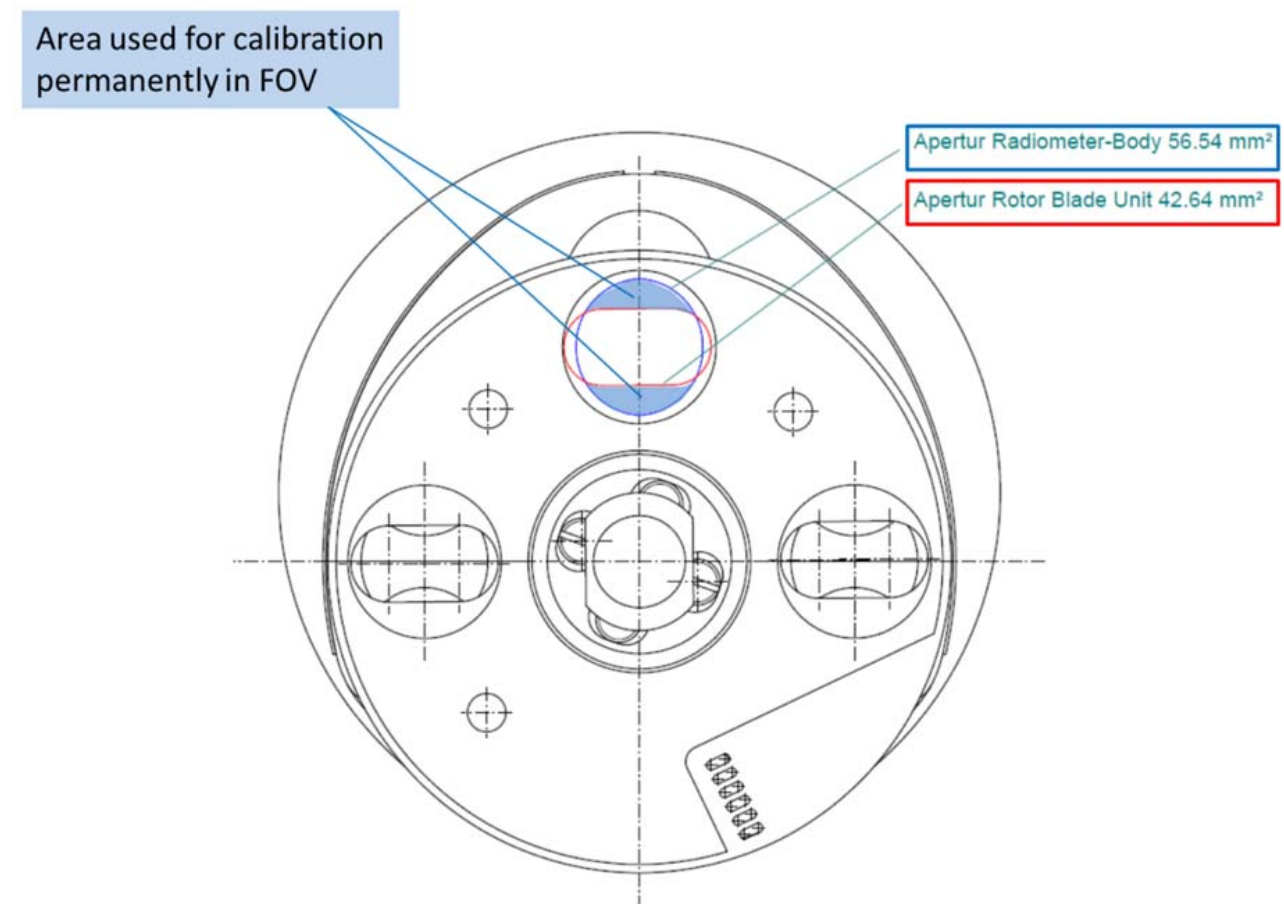

Figure 18 Front view of the calibration target with reduced aperture (red outline) in comparison to the body aperture (blue outline). The temperature of the area marked in blue contributes to the thermopile signal and thus can be used to calibrate the sensors.

\section{SUMMAY AND CONCLUSION}

Although HP3 Radiometer was initially designed on MARA heritage the necessary changes with respect to the different requirements, especially in terms of the compact and very lightweight design, the initial design showed various performance issues. To address this issues an extended redesign of the instrument was necessary. Especially the original concept of sequential open/ close operation of the sensor cover with a DC motor and by this the additional uncontrolled heat emission into the sensor body at opened sensor cover necessitated substantial design changes.

Given by the dimensions of the radiometer, a motor extension by gears of the required size or a space qualified bi-stable solenoid as a alternative option is not available. Therefor the design was changed to the mixed-pixel-concept. For future missions an investigation in alternative options for dust cover movement could improve the instrument performance by refining calibration ability.

In addition the approach of building the sensor body as lightweight as possible in order to have less overall mass had a negative effect on the uniformity of the sensor body temperature distribution. But this is a basic requirement to obtain a high measurement accuracy since the cold junction temperature of the thermopile array has to be known as precisely as possible. 
To achieve higher temperature uniformity the redesign of the sensor body has to have more mass and thereby a higher thermal mass. Furthermore the limitation of temperature sensors for sensor body temperature monitoring to just one sensor limited the accuracy of the temperature control electronics.

A more mature design of the sensor body with respect to temperature measurement, thermal mass and thermal control would also increase the accuracy of the instrument.

The overall design concept of the radiometer an especially the bracket design and manufacturing method are a good foundation for further improvements of the instrument performance and sensitivity. Nevertheless the instrument meets its requirements with respect to mass, size, sensitivity and accuracy.

\section{REFERENCES}

[1] T. Spohn, et al.: "Mupus - a thermal and mechanical properties probe for the Rosetta Lander Philae", Space Sci. Rev. 128(1-4), 339-362 (2007).

[2] H. Hiesinger, J. Helbert et al.: "The Mercury Radiometer and Thermal Infrared Spectrometer (MERTIS) for the BepiColombo mission", Planet. Space Sci. 58(1-2), 144-165 (2010).

[3] J. Gómez-Elvira, C. Armiens, et al.: "REMS: the environmental sensor suite for the Mars science laboratory rover", Space Sci. Rev. 170(1-4), 583-640 (2012).

[4] M. Grott, et al.: "The MASCOT Radiometer MARA for the Hayabusa 2 Mission”, Space Sci. Rev. DOI 10.1007/s11214-016-0272-1 (2016).

[5] Yen, et al.: "An integrated view of the chemistry and mineralogy of martian soils", Nature Volume 436 Issue 7047, 49-54, doi:10.1038/nature03637 (2005).

[6] Ruff, et al.: "The rocks of Gusev Crater as viewed by the Mini-TES instrument", JGR VOL. 111 E12S18, doi:10.1029/2006JE002747 (2006).

[7] Kieffer, et al.: "Thermal model for analysis of Mars infrared mapping”, J. Geophys. Res. Planets 118, 451-470, doi:10.1029/2012JE004164 (2013). 